\title{
Los think tanks en España. Análisis de sus estrategias de comunicación digitales
}

\section{The think tanks in Spain. Analysis of their digital communication strategies}

\author{
Antonio Castillo-Esparcia. Universidad de Málaga. España. \\ acastilloe@uma.es \\ $[\mathrm{CV}] \odot \mathrm{C}$ \\ Elizabet Castillero-Ostio. Universidad de Málaga. España. \\ ecastillero@uma.es \\ $[\mathrm{CV}] \bigcirc \mathrm{G}$ \\ Ana Castillo-Díaz. Universidad de Málaga. España. \\ anacastillo@uma.es \\ $[\mathrm{CV}](\mathrm{C}$
}

Este artículo es producto del proyecto de Investigación titulado "Lobby y Comunicación en España. Análisis de las estrategias de comunicación”, referencia del proyecto CSO2016-79357-R, financiado por el Ministerio de Ciencia, Innovación y Universidades (Programa Estatal de Investigación, Desarrollo e Innovación Orientada a los Retos de la Sociedad).

Cómo citar este artículo / Referencia normalizada

Castillo-Esparcia, A., Castillero-Ostio, E. y Castillo-Díaz, A. (2020). Los think tanks en España. Análisis de sus estrategias de comunicación digitales. Revista Latina de Comunicación Social, (77), 253-273. https://www.doi.org/10.4185/RLCS-2020-1457

\section{RESUMEN}

Introducción: Esta investigación se ocupa de estudiar cómo los think tanks españoles utilizan el medio digital para difundir su actividad y transmitir sus ideas. Metodología: A través del análisis de contenido, se analiza qué tipos de herramientas de comunicación emplean en los espacios digitales que implementan de manera oficial (webs y medios sociales), así como la utilización de estas como canales unidireccionales o bidireccionales. Resultados: Se muestra que los unidireccionales se centran en la difusión de la identidad de estas organizaciones, muy vinculada a las actividades formativas. Entre los bidireccionales, cobran relevancia los medios sociales, que se emplean para la generación de compromiso con sus públicos. Discusión y conclusiones: Los thinks tanks utilizan la comunicación digital como estrategia de difusión de sus actividades. Eso supone potenciar la prospectiva de advocacy y una pretensión de participar en los temas de discusión pública con propuestas sobre sus encuadres (Bürger, 2015, Misztal, 2012).

PALABRAS CLAVE: think tanks; relaciones institucionales; advocacy tanks; comunicación estratégica; comunicación digital.

\section{ABSTRACT}

Introduction: This work focus on the study of how spanish think tanks use digital media to spread their activity and transmit their ideas. Methodology: Through content analysis, we analyze what 
types of communication tools are used in the digital spaces that are officially implemented (web sites and social media), as well as the use of these tools as unidirectional or bidirectional channels. Results: It shows that the unidirectional focus on dissemination of these organizations identities, closely linked to educative activities. Among the bidirectional, social media become relevant, and are used to generate engagement with its stakeholders. Discussion and Conclusions: Think tanks use digital communication as a strategy to disseminate their activities. This fact enhance the prospective of advocacy and a pretension to participate in the topics of public discussion with proposals on its framings (Bürger, 2015, Misztal, 2012).

KEYWORDS: think tanks; institutional relations; advocacy tanks; strategic communication; digital communication.

\section{CONTENIDOS}

1. Introducción. 1.1. Think tanks y comunicación. 2. Metodología. 3. Resultados. 4. Discusión y conclusiones. 5. Referencias.

\section{Introducción}

Hoy en día existe un número reseñable de think tanks con presencia relevante en los procesos políticos y sociales de todo el mundo. La gestión de las ideas ha dejado de ser algo exclusivo de los partidos políticos y los think tanks se perfilan como organizaciones que plantean ideas y soluciones a diversos problemas sociales. Como en todas las organizaciones, sus acciones comunicativas representan un elemento clave en el proceso de relación con sus públicos. Esta faceta es particularmente relevante en la época de los nuevos medios, ya que va a configurar la magnitud de la visibilidad de los think tanks que participen en los actuales procesos de comunicación política.

El objetivo general que persigue este trabajo es el de estudiar cómo los think tanks españoles utilizan el medio digital para difundir su actividad y transmitir sus ideas. En el caso español también se usa la terminología de "laboratorios de ideas". De manera específica, el trabajo analiza qué tipos de herramientas de comunicación se emplean en los distintos espacios digitales que se implementan de manera oficial (sedes webs y medios sociales), así como la utilización de las mismas como canales unidireccionales o bidireccionales en la interacción con sus públicos.

Este acercamiento inicial constituye la base necesaria para establecer una primera aproximación a la gestión de la comunicación de los think tanks y avanzar hacia la optimización de las posibilidades que brindan estos recursos. Estas organizaciones desarrollan estrategias y acciones de comunicación para difundir sus actividades y propuestas temáticas (Almansa-Martínez, Fernández-Torres, 2011).

Desde esta perspectiva, las estrategias de comunicación son elementos esenciales para que toda organización pueda difundir sus actividades y gestionar las relaciones con sus públicos. Hallahan et al. (2007, pp. 3-4) definen la comunicación estratégica como el uso "intencionado de la comunicación, por parte de una organización”.

En su libro, Social Media and Strategic Communications, Al-Deen y Hendricks (2013) apuntan que la publicidad, el marketing y las relaciones públicas se encuentran entre las industrias que lideran la explotación de los medios sociales con fines estratégicos en aras a que puedan alcanzar los objetivos organizativos. 


\subsection{Think tanks y comunicación}

Desde la perspectiva de los think tanks en España asistimos a una multiplicación de estas organizaciones, de sus actividades y de su presencia relevante en los procesos políticos, sociales y comunicativos. La gestión de las ideas circulantes ha dejado de ser espacio exclusivo de los partidos políticos y los think tanks se muestran como organizaciones que proponen ideas, contextos y soluciones a los problemas sociales (Abelson, 2012; Lalueza y Girona, 2016; McGann y Viden Rafferty, 2014; Stone, 1996; Stone y Denham, 2004).

Definidos como centros de investigación o laboratorios de ideas, son organizaciones que realizan investigaciones y análisis sobre la acción política, que generan estudios sobre aspectos concretos y que proponen actuaciones políticas a las instituciones públicas. En este sentido, aportan personal especializado en el análisis y proposición de políticas públicas y son un complemento experto al proceso de elaboración de decisiones institucionales.

McGann (2011) define a los think tanks como organizaciones de investigación, el análisis y la implementación de políticas públicas, que generan estudios, análisis y recomendaciones con respecto a las cuestiones nacionales e internacionales, y que facilitan a los involucrados en la política y a la sociedad en general a tomar decisiones informadas sobre asuntos de política pública.

Estos laboratorios de ideas creadas bajo unas ideas y sin ánimo de lucro, en la mayoría de las ocasiones, son definidos por Castillo (2009) como "entidades que, a través de la investigación y el análisis, proponen propuestas de actuación política a los órganos institucionales mediante estrategias de comunicación directa o indirectas, como es el acceso e influencia sobre la opinión pública". Stone define a los think tanks como: "institutos de investigación independiente cuyo principal objetivo es la investigación de las políticas públicas, [...] organizaciones no gubernamentales sin fines de lucro, independientes del gobierno, partidos políticos y grupo de interés" (1996, p. 16).

Desde una perspectiva histórica, Castillo (2009, pp. 5-6) señala tres períodos principales marcando su desarrollo desde la universidad, pasando por funciones de asesoramiento al gobierno y hasta el activismo en la arena internacional:

- Hasta principios del siglo XX, enmarcados en la investigación universitaria y con un funcionamiento estrictamente investigador, estuvieron centrados en el desarrollo del conocimiento básico.

- A partir de la II Guerra Mundial su participación está relacionada con cuestiones técnicas en el conflicto bélico y será continuada en el marco de la hegemonía estadounidense en la esfera internacional.

- La década de los 70 constituye el escenario de la tercera evolución de estas organizaciones, desarrollándose al amparo la multiplicación de organizaciones sociales nacionales e internacionales.

Los think tanks son organizaciones que pueden implicarse con celeridad y de manera especializada en temas recurrentes de la dinámica política pero también sobre nuevos retos sociales, políticos, geoestratégicos o económicos en los que es necesario disponer del concurso y proposiciones de especialistas en la resolución de los problemas políticos y sociales.

La amplitud conceptual del término abarca a un conjunto de organizaciones con funciones, objetivos y estructuras muy diferenciadas. En este sentido, McGann y Weaver (2000) han estructurado a los think tanks en tres grandes grupos: 
Universities without students cuyo objetivo principal es la publicación de sus investigaciones y con un personal estrictamente investigador. Se diferencian de las universidades en que no imparten formación académica reglada y muchos de ellos se forman bajo la configuración de fundaciones. Poseen una gran variedad de fuentes de financiación como son donaciones de particulares, de empresas, patrocinios y mecenazgos, pero son libertad para acometer las investigaciones más relevantes a sus líneas de interés.

Contract research organization en el que estos laboratorios de ideas realizan investigaciones a partir de un contrato con unos objetivos delimitados y detallados. En este sentido, el modelo sería un contratante (público o privado) que paga por la realización de un servicio determinado.

Advocacy tanks que son aquellos que poseen una ideología, realizan investigaciones sobre cómo resolver los problemas sociales y se convierten en defensores de sus propias ideas. Se caracterizan por actuar para que su ideología impregne las políticas públicas, por lo que desarrollan estrategias de comunicación y tácticas muy activas en forma de informes especializados y seminarios.

Análogamente, Xifra (2003, p. 205) apunta la irrupción de los advocacy tanks que defienden los intereses y ejecutan las acciones políticas más propias de los grupos de interés o lobby y que, muy a menudo, presentan unas u otras vinculaciones ideológicas. Desde la perspectiva de la comunicación, la influencia e impacto, el hecho de perseguir la involucración en el proceso político y objetivos relacionados con advocacy tanks, se convierten en las características propias de los think tanks de finales del siglo XX y principios del siglo XXI (Auger, 2013; Rich y Weaver, 2000; Misztal, 2012; Jan, 2017).

No cabe duda que los laboratorios de ideas desarrollan una serie de actividades que se dirigen hacia el interior del sistema político en forma de asesoramiento, análisis o valoración de políticas públicas ya sea de manera propositiva y/o analítica. Sin embargo, los advocacy tanks tienen una dimensión comunicativa como eje de la difusión de las ideas que defienden estas organizaciones y que desarrollan a través de acciones de educación, sensibilización y legitimación de determinadas posturas sociales (Bürger, 2015; Carim y Warwick, 2013; Ciszek, 2016; Martínez-Salas y Campillo, 2018).

Pero dichas acciones de comunicación no solo se centran en la difusión sino también en el compromiso con sus públicos, que coadyuve a la amplificación de las acciones propositivas de estas organizaciones, difundiendo sus propuestas de manera masiva como prescriptores de la organización (Castillo-Esparcia, García-Ponce y Smolak-Lozano, 2013; Coombs y Holladay, 2015; Gershon, 2016; Kent, 2013).

Todas estas actividades unidireccionales y bidireccionales de comunicación de los think tanks no se diferencian de las estrategias de comunicación de otras organizaciones. Así, planifican, desarrollan y ejecutan campañas de comunicación de sus ideas desde perspectivas diferentes, bajo la consideración de que los diversos públicos deben estar globalmente informados con una pluralidad de canales de comunicación:

- Actividades enfocadas a la dimensión dialógica con los diferentes públicos con los que se relacionan y que se centran en la organización de seminarios o congresos. En ellos, los asistentes son un público ya sensibilizado que acude para conocer las propuestas del think tank o para profundizar en los parámetros de sus iniciativas, convirtiéndose en posibles difusores de estas organizaciones.

- Acciones de difusión en medios de comunicación tradicionales, mediante la organización de comunicados de prensa, ruedas de prensa, entrevistas o artículos de opinión en los medios de 
referencia, entre otros. Con esa actividad mediática persiguen la irradiación a la sociedad de sus propuestas sobre los problemas sociales presentes o el planteamiento de acciones de atención pública. Así, la acción se centra en mantener una presencia opinativa sobre los temas de la agenda pública, una participación activa en el proceso de tematización de determinados asuntos y una proposición de las fronteras conceptuales de la discusión de políticas públicas mediante la función del encuadre.

- Presencia en el ecosistema digital, con la creación de espacios de difusión de sus propuestas que multiplican y generan espacios en plataformas digitales como son redes sociales, blogs o espacios web. Uno de los elementos más relevantes de la sociedad del conocimiento es la multiplicidad de espacios, a través de los que se reproducen las informaciones. En este sentido, mantener una presencia permite reforzar los diferentes mensajes y llegar a otros tipos de públicos que no consumen los medios tradicionales.

- La posibilidad de hacer llegar sus documentos oficiales a cualquiera de sus públicos con la presencia de informes, estudios, valoraciones, libros o documentos de trabajos en el espacio web y que sean de fácil acceso a las personas interesadas.

Todas estas posibilidades de desarrollo comunicativo es una faceta de los think tanks que ha sido señalada por los investigadores y que se debe añadir a la inicial función de asesoramiento y consulta de los poderes públicos en la proposición de propuestas políticas (Stone y Denham, 2004; Xifra, 2003; Abelson, 2012).

Abelson (2000) sostiene que existen varias razones por las cuales los políticos norteamericanos se volcaron y vuelcan sobre los think tanks por información y consejos. En primer lugar, un número importante de estos centros norteamericanos ha desarrollado extensos programas de investigación sobre políticas nacionales e internacionales reclutando no solamente a destacados académicos sino también a ex funcionarios públicos de alto rango. En segundo lugar, los jóvenes profesionales que investigan en los think tanks son más sensibles a lo que los políticos que toman las decisiones necesitan. Les pueden facilitar la información adecuada, concisa y clara para que puedan tomar sus decisiones sabiendo los beneficios asociados a determinadas medidas. En tercer lugar, muchos think tanks pueden proporcionar a los políticos equipos técnicos para cubrir todas las posiciones importantes en el gobierno. En cuarto lugar, los políticos y candidatos a los ministerios pueden buscar a los think tanks para buscar sustento ideológico a sus propuestas.

Además, pueden desarrollar su actividad comunicativa por medio de tres vías distintas: estructurando el debate, modelando de una forma u otra la opinión pública o su clima general y contribuyendo a redefinir o mantener el entendimiento general del interés público.

Este papel parece muy importante, sobre todo en la época de los nuevos medios, ya que va a configurar la magnitud de la visibilidad de los laboratorios de ideas que participen en estos procesos de comunicación política. La contribución de los think tanks al debate público radica en la importancia que poseen los debates públicos para el proceso de decisiones políticas, por lo que los think tanks se pueden considerar como instrumentos potentes de discusión y racionalización de las políticas públicas (Castillo-Esparcia, 2009; McGann, Viden y Rafferty, 2014).

Esta perspectiva configura a los think tanks como organizaciones que ya no solo se centran en interactuar con los organismos gubernamentales. Más allá, se afanan en desarrollar estrategias de comunicación encaminadas a difundir sus análisis y perspectivas, en participar en el proceso social de la construcción del conocimiento y en plantear temas en la agenda pública, proponiendo encuadres específicos en la dinámica interactiva del juego político de las inclusiones, exclusiones y jerarquizaciones de los actores públicos. Son, sin duda, actores políticos en la proposición, 
formulación, análisis y difusión de propuestas de formación y formulación de propuestas políticas a las realidades sociales.

\section{Metodología}

En la presente contribución se plantea un trabajo de carácter exploratorio, cuya principal contribución es establecer el estado actual de la comunicación digital que desarrollan los think tanks españoles.

Este acercamiento inicial se desarrolló mediante un análisis de contenidos de todas las plataformas digitales de los 48 think tanks españoles más influyentes según los trabajos de Tello (2013) y Mcgann (2017). Respecto al listado confeccionado a partir de los propuestos por dichos autores, cabe señalar que se realizó una reorganización, puesto que el número de laboratorios han cambiado en relación a los estudios citados. En la tabla 1 se muestra el listado agrupado según el eje temático en el que se centra su trabajo e indicando el porcentaje que representan sobre el total.

Como puede comprobarse, el mayor porcentaje de think tanks se dedican al ámbito de la economía y las relaciones internacionales, seguidos por los que centran su trabajo en la gobernabilidad, la cultura, la educación y la ciencia.

Tabla 1. Think tanks españoles según su ámbito de trabajo.

\begin{tabular}{|c|c|c|}
\hline $\begin{array}{l}\text { THINK TANKS } \\
\text { ANALIZADOS }\end{array}$ & $\begin{array}{c}\text { PORCENTAJE } \\
\text { SOBRE EL } \\
\text { TOTAL }\end{array}$ & EJES TEMÁTICOS \\
\hline $\begin{array}{l}\text { Barcelona Institute for Global } \\
\text { Health } \\
\text { Basque centre for climate } \\
\text { change } \\
\text { Centro de Desarrollo } \\
\text { Internacional } \\
\text { Ecodes } \\
\text { GRAIN }\end{array}$ & $10.41 \%$ & $\begin{array}{c}\text { Cambio climático/ Agricultura/ Biodiversidad/Gestión eficiente } \\
\text { del agua/ Salud global/ Pobreza energética }\end{array}$ \\
\hline $\begin{array}{l}\text { Círculo de Economía } \\
\text { Círculo de Empresarios } \\
\text { Fundación Alternativas } \\
\text { Fedea } \\
\text { FUNCAS } \\
\text { Fundación Ciudadanía y } \\
\text { Valores } \\
\text { Fundación Estudios Financieros } \\
\text { Institución Futuro } \\
\text { Instituto Estudios Económicos } \\
\text { CIVISMO } \\
\text { Instituto Estudios Fiscales }\end{array}$ & $22.91 \%$ & $\begin{array}{l}\text { Economía/ Política socioeconómica/ Administración Pública/ } \\
\text { Empresa y emprendimiento/ Sociedad/ Bienestar social }\end{array}$ \\
\hline
\end{tabular}




\begin{tabular}{|c|c|c|}
\hline $\begin{array}{l}\text { Centro Internacional de Toledo } \\
\text { para la Paz } \\
\text { CIDOB } \\
\text { Fundación Cultura de Paz } \\
\text { Fundación Euroamérica } \\
\text { Fundación Iberoamérica y } \\
\text { Europa } \\
\text { Iberglobal } \\
\text { IECAH } \\
\text { Instituto Europeo del } \\
\text { Mediterráneo } \\
\text { Real Instituto Elcano } \\
\text { Instituto Juan de Mariana } \\
\text { Seminario de Investigación para } \\
\text { la Paz del Centro Pignatelli }\end{array}$ & $22.91 \%$ & $\begin{array}{l}\text { Relaciones Internacionales/ Paz/ Libertad/ Resolución de } \\
\text { conflictos-mediación/ Política Exterior/ Comercio exterior/ } \\
\text { Cooperación Internacional/ Ayuda humanitaria/ Migraciones }\end{array}$ \\
\hline $\begin{array}{l}\text { Club de Madrid } \\
\text { FAES } \\
\text { Instituto Estudios Democracia } \\
\text { Asociación de Investigación y } \\
\text { Especialización sobre Temas } \\
\text { Iberoamericanos } \\
\text { Centro de Estudios Políticos y } \\
\text { Constitucionales } \\
\text { Instituto Gobernanza } \\
\text { Democrática }\end{array}$ & $12.5 \%$ & $\begin{array}{c}\text { Política/ Gobernanza democrática/ Sociedad inclusiva/ } \\
\text { Humanismo Occidental/ Derechos Humanos/ Gobernabilidad y } \\
\text { participación }\end{array}$ \\
\hline $\begin{array}{l}\text { Fundación Carolina } \\
\text { Fundación Independiente } \\
\text { Fundación de Investigaciones } \\
\text { Marxistas } \\
\text { Fundación Pablo Iglesias } \\
\text { Fundación Ortega y Gasset- } \\
\text { Marañón } \\
\text { Fundación Sistema }\end{array}$ & $12.5 \%$ & $\begin{array}{c}\text { Cultura/ Arte/ Educación/Ciencia/ Ciencias Sociales y Jurídicas/ } \\
\text { Humanidades/Sociedad Civil/ Bioética }\end{array}$ \\
\hline $\begin{array}{l}\text { Grupo de Estudios Estratégicos } \\
\text { (GEES) } \\
\text { Grupo de Estudios sobre } \\
\text { Política y Seguridad } \\
\text { Internacional (GESI) } \\
\text { Instituto Universitario Gutiérrez } \\
\text { Mellado }\end{array}$ & $6.25 \%$ & Seguridad internacional/ Defensa \\
\hline $\begin{array}{l}\text { Cotec } \\
\text { Fundación de la Innovación } \\
\text { Bankinter } \\
\text { Infonomia.com }\end{array}$ & $6.25 \%$ & Innovación/Emprendimiento \\
\hline $\begin{array}{l}\text { Cercle per al Coneixement } \\
\text { (Comisión de SEBAP) } \\
\text { Grupo de Análisis sobre } \\
\text { Estrategia y Prospectiva de la } \\
\text { Información (ThinkEPI) } \\
\text { Observatorio Nacional de las } \\
\text { Telecomunicaciones y de la } \\
\text { Sociedad de la Información }\end{array}$ & $6.25 \%$ & $\begin{array}{c}\text { Tecnologías de la Información y las Comunicaciones (TIC) } \\
\text { Biblioteconomía/Documentación/Lengua española } \\
\text { Sociedad de la Información }\end{array}$ \\
\hline
\end{tabular}

Fuente: elaboración propia.

Para tener conocimiento sobre las herramientas de comunicación digital que utilizan los diferentes think tanks, se toma como punto de partida las páginas web corporativas, que hoy en día constituyen 
el elemento primordial que utilizan las organizaciones para presentarse ante sus públicos. Del contenido que estas ofrezcan y la forma de presentarlo dependerá el interés que susciten entre los visitantes, así como la repercusión que puedan generar estos centros.

Las páginas web de los think tanks estudiados contienen herramientas comunicativas unidireccionales y bidireccionales. Realizando una observación inicial, es posible determinar el nivel de interactividad que presentan estas plataformas digitales oficiales, indicando que las primeras son utilizadas para darse a conocer y difundir sus investigaciones. De manera específica, las segundas, permiten la retroalimentación fluida con sus interlocutores. Entre estas últimas, los medios de comunicación sociales juegan un papel fundamental en cuanto a canal de influencia, dada la intensa actividad que se registra en ellos en nuestros días.

En un primer momento se trató de determinar el tipo de herramienta comunicativa que se estaba utilizando en los distintos portales web (unidireccional o bidireccional). Posteriormente, se identificó la tipología de contenido difundido, así como la frecuencia de actualización del mismo que publicaban los diversos think tanks en sus medios sociales oficiales. Para esta tarea se recurrió al análisis de contenido. El tiempo de análisis acotado fue de un mes, desde el 1 al 30 de abril de 2018, considerando este intervalo como adecuado para observar la actividad comunicativa de estas organizaciones en el dinámico entorno digital.

Con la finalidad de categorizar las herramientas comunicativas se diseñó una plantilla de análisis (tabla 2) que incluye todas las herramientas de comunicación registradas en las plataformas de comunicación oficial de los think tanks españoles.

Tabla 2. Plantilla de análisis para páginas web corporativas de think tanks.

\begin{tabular}{|c|c|}
\hline $\begin{array}{l}\text { HERRAMIENTAS DE } \\
\text { COMUNICACIÓN } \\
\text { UNIDIRECCIONALES }\end{array}$ & $\begin{array}{c}\text { HERRAMIENTAS DE COMUNICACIÓN } \\
\text { BIDIRECCIONALES }\end{array}$ \\
\hline $\begin{array}{l}\text { Archivos multimedia insertados en página } \\
\text { web (audios, vídeos) }\end{array}$ & Ruedas de prensa \\
\hline Fotografías e imágenes & Entrevistas \\
\hline Sala de prensa virtual (Comunicados) & Seminarios/ Conferencias/ Congresos \\
\hline Noticias publicadas en la web & Jornadas/ Talleres \\
\hline $\begin{array}{c}\text { Publicaciones: Estudios, informes } \\
\text { temáticos, libros, artículos, publicaciones } \\
\text { periódicas (Revistas) }\end{array}$ & Simposios y mesas redondas \\
\hline Boletín informativo (Newsletter) & Intervenciones en actos académicos \\
\hline $\begin{array}{l}\text { Folleto informativo (Presentación del Think } \\
\text { tank) }\end{array}$ & Encuentros con expertos \\
\hline Anuarios institucionales (Memorias) & Otros eventos con orientación formativa/educación \\
\hline Campañas en medios de comunicación & Cursos y posgrados \\
\hline \multirow[t]{6}{*}{ Publicación de agenda de eventos } & Otros eventos (fiestas, premios) \\
\hline & Foros (sitios de discusión en línea) \\
\hline & Blogs \\
\hline & Campus virtual \\
\hline & Intranet \\
\hline & Redes sociales \\
\hline
\end{tabular}

Fuente: elaboración propia. 
En cada caso, se identificó la temática del contenido difundido a través de las distintas herramientas empleadas.

Por otra parte, como se ha señalado, los medios sociales (redes y blogs) constituyen recursos especialmente relevantes en cuanto a su nivel de uso, debido en buena medida a las posibilidades de interacción que ofrecen. En este sentido, se diseñó una plantilla específica (tabla 3) para realizar un análisis de la actividad desarrollada en estos espacios. Como se puede apreciar, la lista incluye los medios sociales en los que los think tanks españoles tienen una participación oficial significativa en cuanto a creación de contenidos (Facebook, Twitter, YouTube y blogs) y recoge información sobre la temática y la actualización de los contenidos (creados o compartidos).

Tabla 3. Plantilla de análisis de medios sociales de think tanks.

\begin{tabular}{|c|l|}
\hline MEDIOS SOCIALES ANALIZADOS & \multicolumn{1}{c|}{ ASPECTOS OBSERVADOS } \\
\hline Facebook & $\begin{array}{l}\text { Frecuencia actualización contenido creado } \\
\text { Frecuencia contenido compartido } \\
\text { Temática de las entradas }\end{array}$ \\
\hline Twitter & $\begin{array}{l}\text { Frecuencia actualización } \\
\text { Frecuencia retuit } \\
\text { Temática de las entradas }\end{array}$ \\
\hline YouTube & $\begin{array}{l}\text { Frecuencia actualización } \\
\text { Temática de las entradas }\end{array}$ \\
\hline Blogs & $\begin{array}{l}\text { Frecuencia actualización } \\
\text { Temática de las entradas }\end{array}$ \\
\hline
\end{tabular}

Fuente: elaboración propia.

\section{Resultados}

Por una parte, en el análisis se abordan las herramientas de comunicación unidireccionales que tratan de establecer una conexión con el visitante web con el objetivo de mostrar información corporativa, permitiendo que, tanto los medios de comunicación como la ciudadanía, estén informados sobre su identidad y actividades.

En este grupo de herramientas (tabla 4) se encuentran aquellas que permiten la presentación del organismo (credo fundacional, objetivos, expertos y equipos de investigación), la difusión de sus investigaciones, eventos, campañas comunicativas desarrolladas y otras noticias. Esta información se complementa a través de la publicación de boletines informativos, anuarios/memorias, agendas de eventos o folletos. Más allá de estos recursos genéricos, existen espacios destinados a la relación con los medios de comunicación, fundamentalmente denominados como sala de prensa virtual.

Todos los think tanks españoles disponen de algunas de las herramientas mostradas en la tabla 4 en sus respectivas webs. Porcentualmente, destacan en primer lugar las publicaciones que reflejan estudios, informes temáticos, libros, artículos, así como las publicaciones periódicas (revistas), ya que el $100 \%$ de la muestra dispone de este tipo de herramienta unidireccional. Le siguen en frecuencia de utilización las fotografías e imágenes, en tercer lugar, los archivos multimedia, las noticias y los anuarios o memorias institucionales. La organización que cuenta con más recursos unidireccionales es el Barcelona Institute for Global Health, seguida de CIDOB. 
RLCS, Revista Latina de Comunicación Social, 77, 253-273

[Investigación] DOI: 10.4185/RLCS-2020-1457 | ISSN 1138-5820 | Año 2020

Tabla 4. Herramientas de comunicación unidireccionales en la página web.

\begin{tabular}{|c|c|c|c|c|c|c|c|c|c|c|}
\hline \multirow{2}{*}{$\begin{array}{l}\text { THINK TANKS } \\
\text { ANALIZADOS }\end{array}$} & \multicolumn{10}{|c|}{ HERRAMIENTAS DE COMUNICACIÓN UNIDIRECCIONALES } \\
\hline & 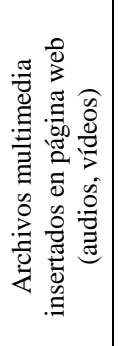 & 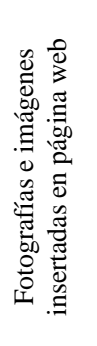 & 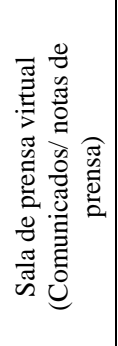 & 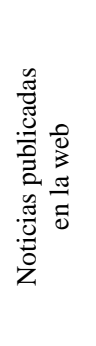 & 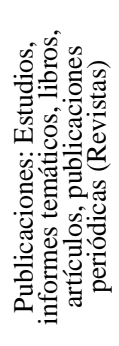 & 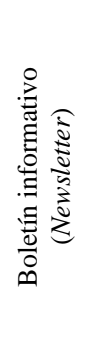 & 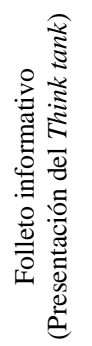 & 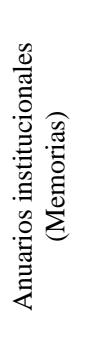 & 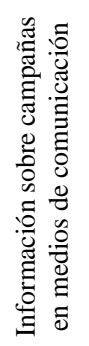 & 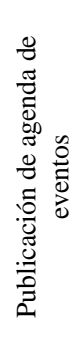 \\
\hline $\begin{array}{l}\text { Barcelona Institute for Global } \\
\text { Health }\end{array}$ & $\mathrm{X}$ & $\mathrm{X}$ & $\mathrm{X}$ & $\mathrm{X}$ & $\mathrm{X}$ & $\mathrm{X}$ & $\mathrm{X}$ & $\mathrm{X}$ & $\mathrm{X}$ & $\mathrm{X}$ \\
\hline CIDOB & $\mathrm{X}$ & $\mathrm{X}$ & $\mathrm{X}$ & $\mathrm{X}$ & $\mathrm{X}$ & $\mathrm{X}$ & $\mathrm{X}$ & $\mathrm{X}$ & & $\mathrm{X}$ \\
\hline $\begin{array}{l}\text { Círculo de Empresarios/ } \\
\text { Cotec/ F. Alternativas/ } \\
\text { FUNCAS/ Fundación de la } \\
\text { Innovación Bankinter/ } \\
\text { Fundación Pablo Iglesias / } \\
\text { Fundación Ortega y Gasset- } \\
\text { Marañón / IECAH/ Institución } \\
\text { Futuro/ Instituto Estudios } \\
\text { Democracia/ Instituto Europeo } \\
\text { del Mediterráneo/ Real } \\
\text { Instituto Elcano/ Cercle per al } \\
\text { Coneixement (Comisión de } \\
\text { SEBAP) }\end{array}$ & $\mathrm{X}$ & $\mathrm{X}$ & $\mathrm{X}$ & $\mathrm{X}$ & $\mathrm{X}$ & $\mathrm{X}$ & & $\mathrm{X}$ & & $\mathrm{X}$ \\
\hline Ecodes/ GRAIN & $\mathrm{X}$ & $\mathrm{X}$ & $\mathrm{X}$ & $\mathrm{X}$ & $\mathrm{X}$ & $\mathrm{X}$ & & $\mathrm{X}$ & $\mathrm{X}$ & \\
\hline FAES/ F. Cultura de Paz & $\mathrm{X}$ & $\mathrm{X}$ & $\mathrm{X}$ & $\mathrm{X}$ & $\mathrm{X}$ & $\mathrm{X}$ & & $\mathrm{X}$ & & \\
\hline $\begin{array}{l}\text { CIVISMO/ Instituto Juan de } \\
\text { Mariana }\end{array}$ & $\mathrm{X}$ & $\mathrm{X}$ & $\mathrm{X}$ & $\mathrm{X}$ & $\mathrm{X}$ & $\mathrm{X}$ & & & & $\mathrm{X}$ \\
\hline $\begin{array}{l}\text { Centro de Estudios Políticos y } \\
\text { Constitucionales }\end{array}$ & $\mathrm{X}$ & $\mathrm{X}$ & $\mathrm{X}$ & $\mathrm{X}$ & $\mathrm{X}$ & & & $\mathrm{X}$ & & $\mathrm{X}$ \\
\hline $\begin{array}{l}\text { Grupo de Análisis sobre } \\
\text { Estrategia y Prospectiva de la } \\
\text { Información (ThinkEPI) }\end{array}$ & $\mathrm{X}$ & $\mathrm{X}$ & & $\mathrm{X}$ & $\mathrm{X}$ & $\mathrm{X}$ & & $\mathrm{X}$ & & $\mathrm{X}$ \\
\hline $\begin{array}{l}\text { Asociación de Investigación y } \\
\text { Especialización sobre Temas } \\
\text { Iberoamericanos }\end{array}$ & $\mathrm{X}$ & $\mathrm{X}$ & & $\mathrm{X}$ & $\mathrm{X}$ & $\mathrm{X}$ & & $\mathrm{X}$ & $\mathrm{X}$ & \\
\hline $\begin{array}{l}\text { Basque centre for climate } \\
\text { change/ Instituto Estudios } \\
\text { Económicos }\end{array}$ & $\mathrm{X}$ & $\mathrm{X}$ & $\mathrm{X}$ & $\mathrm{X}$ & $\mathrm{X}$ & & & $\mathrm{X}$ & & \\
\hline Fundación Independiente & $\mathrm{X}$ & $\mathrm{X}$ & $\mathrm{X}$ & $\mathrm{X}$ & $\mathrm{X}$ & & & & & $\mathrm{X}$ \\
\hline $\begin{array}{l}\text { Centro de Desarrollo } \\
\text { Internacional, Universidad de } \\
\text { Navarra }\end{array}$ & $\mathrm{X}$ & $\mathrm{X}$ & $X$ & $\mathrm{X}$ & $\mathrm{X}$ & & & & & $\mathrm{X}$ \\
\hline $\begin{array}{l}\text { Instituto Gobernanza } \\
\text { Democrática / F. Estudios } \\
\text { Financieros }\end{array}$ & $\mathrm{X}$ & $\mathrm{X}$ & & $\mathrm{X}$ & $\mathrm{X}$ & & & $\mathrm{X}$ & & $\mathrm{X}$ \\
\hline $\begin{array}{l}\text { Instituto Universitario } \\
\text { Gutiérrez Mellado }\end{array}$ & $\mathrm{X}$ & $\mathrm{X}$ & & $\mathrm{X}$ & $\mathrm{X}$ & $\mathrm{X}$ & & $\mathrm{X}$ & & \\
\hline Fundación Carolina & $\mathrm{X}$ & $X$ & $\mathrm{X}$ & $\mathrm{X}$ & $\mathrm{X}$ & & & & & \\
\hline $\begin{array}{l}\text { Centro Internacional de } \\
\text { Toledo para la Paz }\end{array}$ & $\mathrm{X}$ & $\mathrm{X}$ & & & $\mathrm{X}$ & & & $\mathrm{X}$ & & $\mathrm{X}$ \\
\hline $\begin{array}{l}\text { Fundación Ciudadanía y } \\
\text { Valores }\end{array}$ & & $\mathrm{X}$ & & $\mathrm{X}$ & $\mathrm{X}$ & $\mathrm{X}$ & & & & $\mathrm{X}$ \\
\hline
\end{tabular}


RLCS, Revista Latina de Comunicación Social, 77, 253-273

[Investigación] DOI: 10.4185/RLCS-2020-1457 | ISSN 1138-5820 | Año 2020

\begin{tabular}{|c|c|c|c|c|c|c|c|c|c|c|}
\hline F. Euroamérica & $\mathrm{X}$ & $\mathrm{X}$ & & $\mathrm{X}$ & $\mathrm{X}$ & & & & & \\
\hline $\begin{array}{l}\text { Fundación de Investigaciones } \\
\text { Marxistas }\end{array}$ & $\mathrm{X}$ & $\mathrm{X}$ & & $\mathrm{X}$ & $\mathrm{X}$ & & & $\mathrm{X}$ & & \\
\hline Fedea & $\mathrm{X}$ & $\mathrm{X}$ & $\mathrm{X}$ & & $\mathrm{X}$ & & & & & $\mathrm{X}$ \\
\hline $\begin{array}{l}\text { Observatorio Nacional de las } \\
\text { Telecomunicaciones y de la } \\
\text { Sociedad de la Información }\end{array}$ & $\mathrm{X}$ & $\mathrm{X}$ & & & $\mathrm{X}$ & $\mathrm{X}$ & & $\mathrm{X}$ & & \\
\hline Fundación Sistema & $\mathrm{X}$ & $\mathrm{X}$ & & $\mathrm{X}$ & $\mathrm{X}$ & $\mathrm{X}$ & & & & \\
\hline Círculo de Economía & $\mathrm{X}$ & $\mathrm{X}$ & & & $\mathrm{X}$ & & & $\mathrm{X}$ & & \\
\hline $\begin{array}{l}\text { Fundación Iberoamérica y } \\
\text { Europa }\end{array}$ & & $\mathrm{X}$ & $\mathrm{X}$ & $\mathrm{X}$ & $\mathrm{X}$ & & & & & \\
\hline Instituto Estudios Fiscales & & & & $\mathrm{X}$ & $\mathrm{X}$ & & & $\mathrm{X}$ & & $\mathrm{X}$ \\
\hline $\begin{array}{l}\text { Seminario de Investigación } \\
\text { para la Paz del Centro } \\
\text { Pignatelli }\end{array}$ & & $\mathrm{X}$ & & & $\mathrm{X}$ & $\mathrm{X}$ & & & & $\mathrm{X}$ \\
\hline $\begin{array}{l}\text { Infonomia.com/ Grupo de } \\
\text { Estudios sobre Política y } \\
\text { Seguridad Internacional } \\
\text { (GESI) }\end{array}$ & $\mathrm{X}$ & $\mathrm{X}$ & & & $\mathrm{X}$ & & & & & \\
\hline Club de Madrid & & $\mathrm{X}$ & & $\mathrm{X}$ & $\mathrm{X}$ & & & & & \\
\hline $\begin{array}{l}\text { GEES (Grupo de Estudios } \\
\text { Estratégicos) / Iberglobal }\end{array}$ & & & & & $\mathrm{X}$ & $\mathrm{X}$ & & & & \\
\hline TOTAL & 41 & 46 & 29 & 39 & 48 & 31 & 2 & 32 & 4 & 28 \\
\hline PORCENTAJE (\%) & 85,41 & 95,83 & 60,41 & 81,25 & 100 & 64,58 & 4,16 & 66,66 & 8,33 & 58,33 \\
\hline
\end{tabular}

Fuente: elaboración propia.

En lo que respecta al análisis de las herramientas de comunicación bidireccionales (tabla 5), todos los think tanks disponen de perfiles en redes sociales. Un porcentaje acusado de ellos $(81,25 \%)$ organizan seminarios, congresos, conferencias, jornadas y talleres cuyos contenidos difunden a través de sus plataformas digitales. Asimismo, se registran porcentajes elevados de aquellos que incluyen actividades formativas (50\%) y aquellos que desarrollan blogs (43,75\%). Al igual que en el caso de las herramientas unidireccionales, el Barcelona Institute for Global Health destaca en cuanto a organización de actividades, entre las que sobresalen mesas redondas, debates abiertos, entrevistas a expertos e investigadores y jornadas.

Teniendo en cuenta el tipo de actividades que se difunden a través de estos recursos, sobresalen aquellas de carácter educativo. Es el caso de las jornadas, cursos, seminarios, proyectos o la convocatoria de becas, prácticas y premios en materia de educación. En ocasiones, para la gestión de la actividad formativa, los think tanks organizan o participan en institutos de investigación, implementan universidades de mayores (el caso de la Fundación José Ortega y Gasset-Gregorio Marañón), así como desarrollan escuelas de verano o programas de intercambio (Basque centre for climate change, CIVISMO, Fundación de Investigaciones Marxistas, FAES y el Instituto Estudios Fiscales). Gran parte de ellos imparten cursos, seminarios y posgrados o participan en la docencia universitaria de otros centros. 
RLCS, Revista Latina de Comunicación Social, 77, 253-273

[Investigación] DOI: 10.4185/RLCS-2020-1457 | ISSN 1138-5820 | Año 2020

Tabla 5. Herramientas de comunicación bidireccionales en las páginas web de los think tanks españoles.

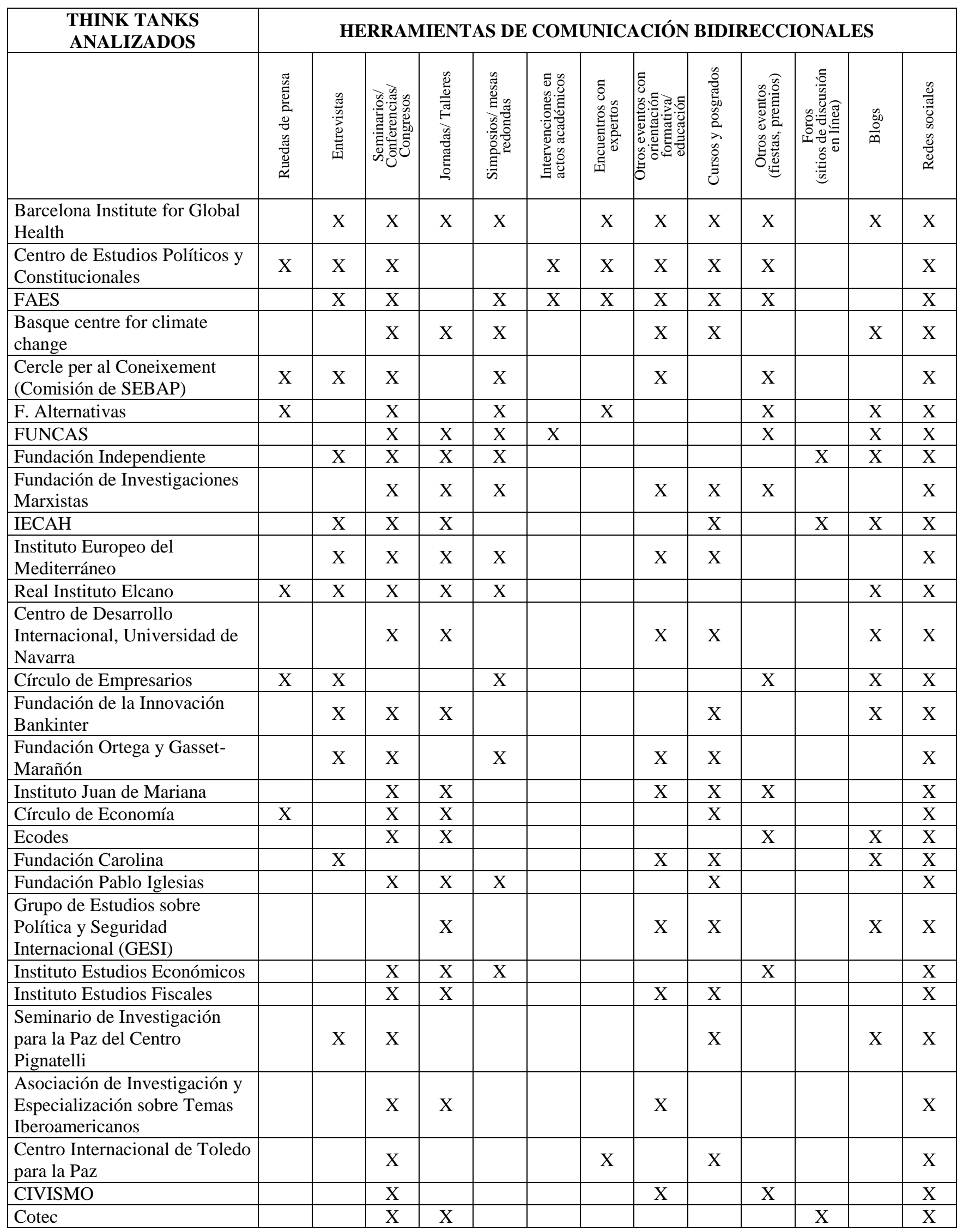


RLCS, Revista Latina de Comunicación Social, 77, 253-273

[Investigación] DOI: 10.4185/RLCS-2020-1457 | ISSN 1138-5820 | Año 2020

\begin{tabular}{|c|c|c|c|c|c|c|c|c|c|c|c|c|c|}
\hline $\begin{array}{l}\text { Fundación Ciudadanía y } \\
\text { Valores }\end{array}$ & & & $\mathrm{X}$ & & & & & & $\mathrm{X}$ & & & $\mathrm{X}$ & $\mathrm{X}$ \\
\hline F. Cultura de Paz & & $\mathrm{X}$ & $\mathrm{X}$ & & & & & & & & & $\mathrm{X}$ & $\mathrm{X}$ \\
\hline $\begin{array}{l}\text { Grupo de Análisis sobre } \\
\text { Estrategia y Prospectiva de la } \\
\text { Información (ThinkEPI) }\end{array}$ & & & $\mathrm{X}$ & $\mathrm{X}$ & & & & & & & & $\mathrm{X}$ & $X$ \\
\hline Institución Futuro & & & $\mathrm{X}$ & $\mathrm{X}$ & & & & & & & & $\mathrm{X}$ & $\mathrm{X}$ \\
\hline $\begin{array}{l}\text { Instituto Gobernanza } \\
\text { Democrática }\end{array}$ & & & $\mathrm{X}$ & $\mathrm{X}$ & & & & $\mathrm{X}$ & & & & & $\mathrm{X}$ \\
\hline $\begin{array}{l}\text { Instituto Universitario } \\
\text { Gutiérrez Mellado }\end{array}$ & & $\mathrm{X}$ & $\mathrm{X}$ & & & & & & $\mathrm{X}$ & & & & $X$ \\
\hline CIDOB & & $\mathrm{X}$ & $\mathrm{X}$ & & & & & & & & & & $\mathrm{X}$ \\
\hline Fedea & & & $\mathrm{X}$ & $\mathrm{X}$ & & & & & & & & & $\mathrm{X}$ \\
\hline F. Estudios Financieros & & & $\mathrm{X}$ & & & & & & $\mathrm{X}$ & & & & $\mathrm{X}$ \\
\hline F. Euroamérica & & & $\mathrm{X}$ & & & & & $\mathrm{X}$ & & & & & $\mathrm{X}$ \\
\hline $\begin{array}{l}\text { Fundación Iberoamérica y } \\
\text { Europa }\end{array}$ & & & $\mathrm{X}$ & & & & & & $\mathrm{X}$ & & & & $\mathrm{X}$ \\
\hline GRAIN & & $\mathrm{X}$ & & & & & & & & & & $\mathrm{X}$ & $\mathrm{X}$ \\
\hline Iberglobal & & & & & & & & & $\mathrm{X}$ & & & $\mathrm{X}$ & $\mathrm{X}$ \\
\hline Instituto Estudios Democracia & & & $\mathrm{X}$ & & & & & & $\mathrm{X}$ & & & & $\mathrm{X}$ \\
\hline Fundación Sistema & & & $\mathrm{X}$ & & & & & & & & & & $\mathrm{X}$ \\
\hline Infonomia.com & & & & & & & & & & & & $X$ & $\mathrm{X}$ \\
\hline Club de Madrid & & & & & & & & & & & & & $\mathrm{X}$ \\
\hline $\begin{array}{l}\text { GEES (Grupo de Estudios } \\
\text { Estratégicos) }\end{array}$ & & & & & & & & & & & & & $X$ \\
\hline $\begin{array}{l}\text { Observatorio Nacional de las } \\
\text { Telecomunicaciones y de la } \\
\text { Sociedad de la Información }\end{array}$ & & & & & & & & & & & & & $X$ \\
\hline TOTAL & 6 & 17 & 39 & 23 & 14 & 3 & 5 & 17 & 24 & 12 & 3 & 21 & 48 \\
\hline PORCENTAJE $(\%)$ & 12,53 & 35,42 & 81,25 & 47,92 & 29,17 & 6,25 & 10,42 & 35,42 & 50 & 25 & 6,25 & 43,75 & 100 \\
\hline
\end{tabular}

Fuente: elaboración propia.

Teniendo en cuenta la utilización por parte de los think tanks de las herramientas comunicativas, tanto unidireccionales como bidireccionales, se ha elaborado un ranking (tabla 6) que muestra a los centros más y menos comunicativos, según la información reflejada en sus portales en el medio digital.

En este ranking se puede observar, si tenemos en cuenta la doble tipología de herramientas comunicativas, que lo encabeza el Barcelona Institute for Global Health, que utiliza el 91,30\% de las categorías tenidas en cuenta. Le siguen, empatados, el Centro de Estudios Políticos y Constitucionales y FAES con el 69,56\% de la utilización de estas herramientas. Cierran el listado, como los think tanks que menos muestran acciones comunicativas con sus diferentes públicos, el Club de Madrid y el GEES (Grupo de Estudios Estratégicos).

Tabla 6. Ranking de los think tanks españoles más comunicativos según la información reflejada en el medio digital.

\begin{tabular}{|l|l|c|c|c|}
\hline & \multicolumn{1}{|c|}{$\begin{array}{c}\text { RANKING DE LOS THINK TANKS MÁS } \\
\text { COMUNICATIVOS } \\
\text { SEGÚN LA INFORMACIÓN REFLEJADA EN } \\
\text { EL MEDIO DIGITAL }\end{array}$} & $\begin{array}{c}\mathbf{N}^{\mathbf{0}} \\
\text { Herramientas } \\
\text { unidireccionales } \\
\text { utilizadas }\end{array}$ & $\begin{array}{c}\mathbf{N}^{\mathbf{1}} \\
\text { Herramientas } \\
\text { bidireccionales } \\
\text { utilizadas }\end{array}$ & $\begin{array}{c}\text { Total } \\
\text { herramientas } \\
\text { utilizadas }\end{array}$ \\
\hline 1. & Barcelona Institute for Global Health & 10 & 10 & 20 \\
\hline \multirow{2}{*}{2.} & Centro de Estudios Políticos y Constitucionales & 7 & 9 & 16 \\
\cline { 2 - 5 } & FAES & 7 & 9 & 16 \\
\hline
\end{tabular}


RLCS, Revista Latina de Comunicación Social, 77, 253-273

[Investigación] DOI: 10.4185/RLCS-2020-1457 | ISSN 1138-5820 | Año 2020

\begin{tabular}{|c|c|c|c|c|}
\hline \multirow{6}{*}{3.} & Cercle per al Coneixement (Comisión de SEBAP) & 8 & 7 & 15 \\
\hline & F. Alternativas & 8 & 7 & 15 \\
\hline & FUNCAS & 8 & 7 & 15 \\
\hline & IECAH & 8 & 7 & 15 \\
\hline & Instituto Europeo del Mediterráneo & 8 & 7 & 15 \\
\hline & Real Instituto Elcano & 8 & 7 & 15 \\
\hline \multirow{3}{*}{4.} & Círculo de Empresarios & 8 & 6 & 14 \\
\hline & Fundación de la Innovación Bankinter & 8 & 6 & 14 \\
\hline & Fundación Ortega y Gasset-Marañón & 8 & 6 & 14 \\
\hline \multirow{5}{*}{5.} & Basque centre for climate change & 6 & 7 & 13 \\
\hline & Fundación Independiente & 6 & 7 & 13 \\
\hline & Instituto Juan de Mariana & 7 & 6 & 13 \\
\hline & Ecodes & 8 & 5 & 13 \\
\hline & Fundación Pablo Iglesias & 8 & 5 & 13 \\
\hline \multirow{5}{*}{6.} & Fundación de Investigaciones Marxistas & 5 & 7 & 12 \\
\hline & $\begin{array}{l}\text { Centro de Desarrollo Internacional, Universidad de } \\
\text { Navarra }\end{array}$ & 6 & 6 & 12 \\
\hline & Cotec & 8 & 4 & 12 \\
\hline & Institución Futuro & 8 & 4 & 12 \\
\hline & CIDOB & 9 & 3 & 12 \\
\hline \multirow{7}{*}{7.} & Instituto Estudios Económicos & 6 & 5 & 11 \\
\hline & $\begin{array}{l}\text { Asociación de Investigación y Especialización sobre } \\
\text { Temas Iberoamericanos }\end{array}$ & 7 & 4 & 11 \\
\hline & CIVISMO & 7 & 4 & 11 \\
\hline & F. Cultura de Paz & 7 & 4 & 11 \\
\hline & $\begin{array}{l}\text { Grupo de Análisis sobre Estrategia y Prospectiva de } \\
\text { la Información (ThinkEPI) }\end{array}$ & 7 & 4 & 11 \\
\hline & GRAIN & 8 & 3 & 11 \\
\hline & Instituto Estudios Democracia & 8 & 3 & 11 \\
\hline \multirow{5}{*}{8.} & Fundación Carolina & 5 & 5 & 10 \\
\hline & $\begin{array}{l}\text { Seminario de Investigación para la Paz del Centro } \\
\text { Pignatelli }\end{array}$ & 5 & 5 & 10 \\
\hline & Instituto Gobernanza Democrática & 6 & 4 & 10 \\
\hline & Instituto Universitario Gutiérrez Mellado & 6 & 4 & 10 \\
\hline & F. Estudios Financieros & 7 & 3 & 10 \\
\hline \multirow{5}{*}{9.} & Círculo de Economía & 4 & 5 & 9 \\
\hline & Instituto Estudios Fiscales & 4 & 5 & 9 \\
\hline & Centro Internacional de Toledo para la Paz & 5 & 4 & 9 \\
\hline & Fundación Ciudadanía y Valores & 5 & 4 & 9 \\
\hline & Fedea & 6 & 3 & 9 \\
\hline \multirow[t]{2}{*}{10.} & $\begin{array}{l}\text { Grupo de Estudios sobre Política y Seguridad } \\
\text { Internacional (GESI) }\end{array}$ & 3 & 5 & 8 \\
\hline & F. Euroamérica & 5 & 3 & 8 \\
\hline \multirow{3}{*}{11.} & Fundación Iberoamérica y Europa & 4 & 3 & 7 \\
\hline & Fundación Sistema & 5 & 2 & 7 \\
\hline & $\begin{array}{l}\text { Observatorio Nacional de las Telecomunicaciones y } \\
\text { de la Sociedad de la Información }\end{array}$ & 6 & 1 & 7 \\
\hline \multirow{2}{*}{12.} & Iberglobal & 2 & 3 & 5 \\
\hline & Infonomia.com & 3 & 2 & 5 \\
\hline 13. & Club de Madrid & 3 & 1 & 4 \\
\hline 14. & GEES (Grupo de Estudios Estratégicos) & 2 & 1 & 3 \\
\hline
\end{tabular}

Fuente: elaboración propia.

Tal como se ha indicado en la revisión bibliográfica, la comunicación de los think tanks a través de medios sociales juega un papel determinante en la actualidad. De hecho, el 100\% de think tanks los 
RLCS, Revista Latina de Comunicación Social, 77, 253-273

[Investigación] DOI: 10.4185/RLCS-2020-1457 | ISSN 1138-5820 | Año 2020

utilizan. Un primer análisis de los resultados reflejados en la tabla 7, permite afirmar que Twitter y Facebook son las redes sociales más empleadas. De esta forma un 87,50\% de las organizaciones estudiadas usan Twitter. Con cifras ligeramente por debajo $(85,41 \%)$ le sigue Facebook. En tercer lugar, se encuentra LinkedIn, un $64,58 \%$ del total usan esta red para aglutinar a diferentes profesionales o crear grupos donde comparten intereses. También destaca la presencia de estos centros en YouTube $(62,5 \%)$. Menos significativa es la apuesta realizada en plataformas como Google+(45,83\%) o redes de fotografías como Flickr $(39,58 \%)$. En los últimos puestos de este análisis estarían aquellas organizaciones que tienen presencia en Instagram $(22,91 \%)$, Vimeo y Pinterest $(27,08 \%$ en su conjunto). En cuanto al uso de redes sociales, la Fundación Bankinter, seguida por el Barcelona Institute for Global Health son los think tanks más activos.

Tabla 7. Presencia global de los think tanks españoles en las redes sociales.

\begin{tabular}{|c|c|c|c|c|c|c|c|c|c|}
\hline Think tanks & 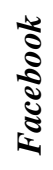 & 氮 & 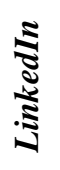 & 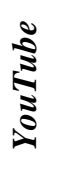 & 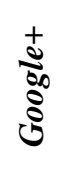 & 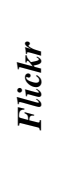 & 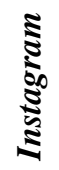 & $\stackrel{\mathscr{0}}{0}$ & 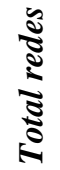 \\
\hline $\begin{array}{l}\text { Fundación de la Innovación } \\
\text { Bankinter }\end{array}$ & $\mathrm{X}$ & $\mathrm{X}$ & $\mathrm{X}$ & $\mathrm{X}$ & $\mathrm{X}$ & $\mathrm{X}$ & $\mathrm{X}$ & Pinterest/Vimeo & 9 \\
\hline $\begin{array}{l}\text { Barcelona Institute for Global } \\
\text { Health }\end{array}$ & $\mathrm{X}$ & $\mathrm{X}$ & $\mathrm{X}$ & $X$ & $\mathrm{X}$ & $\mathrm{X}$ & $\mathrm{X}$ & Vimeo & 8 \\
\hline Círculo de Empresarios & $\mathrm{X}$ & $\mathrm{X}$ & $\mathrm{X}$ & $\mathrm{X}$ & $X$ & $\mathrm{X}$ & $\mathrm{X}$ & Pinterest & 7 \\
\hline Institución Futuro & $\mathrm{X}$ & $\mathrm{X}$ & $\mathrm{X}$ & $\mathrm{X}$ & $\mathrm{X}$ & $\mathrm{X}$ & & Vimeo & 7 \\
\hline Infonomia.com & $\mathrm{X}$ & $\mathrm{X}$ & $\mathrm{X}$ & $\mathrm{X}$ & $\mathrm{X}$ & $\mathrm{X}$ & $\mathrm{X}$ & & 7 \\
\hline Real Instituto Elcano & $\mathrm{X}$ & $\mathrm{X}$ & $\mathrm{X}$ & $\mathrm{X}$ & $\mathrm{X}$ & $\mathrm{X}$ & $\mathrm{X}$ & & 7 \\
\hline CIVISMO & $\mathrm{X}$ & $\mathrm{X}$ & $\mathrm{X}$ & $\mathrm{X}$ & $\mathrm{X}$ & $\mathrm{X}$ & $\mathrm{X}$ & & 6 \\
\hline Fundación Alternativas & $\mathrm{X}$ & $\mathrm{X}$ & $\mathrm{X}$ & $\mathrm{X}$ & & $\mathrm{X}$ & & Vimeo & 6 \\
\hline Fundación Carolina & $\mathrm{X}$ & $\mathrm{X}$ & $\mathrm{X}$ & $\mathrm{X}$ & & $\mathrm{X}$ & $\mathrm{X}$ & & 6 \\
\hline $\begin{array}{l}\text { Fundación Ciudadanía y } \\
\text { Valores }\end{array}$ & $\mathrm{X}$ & $\mathrm{X}$ & X & $\mathrm{X}$ & $\mathrm{X}$ & & & Vimeo & 6 \\
\hline CIDOB & $\mathrm{X}$ & $X$ & $X$ & $X$ & $\mathrm{X}$ & & $\mathrm{X}$ & & 5 \\
\hline Club de Madrid & $\mathrm{X}$ & $\mathrm{X}$ & $\mathrm{X}$ & $\mathrm{X}$ & $\mathrm{X}$ & $\mathrm{X}$ & & & 5 \\
\hline Cotec & $\mathrm{X}$ & $X$ & $\mathrm{X}$ & $\mathrm{X}$ & $\mathrm{X}$ & $\mathrm{X}$ & $\mathrm{X}$ & & 5 \\
\hline $\begin{array}{l}\text { Fundación Estudios } \\
\text { Financieros }\end{array}$ & $\mathrm{X}$ & $\mathrm{X}$ & $\mathrm{X}$ & $\mathrm{X}$ & & & & Vimeo & 5 \\
\hline $\begin{array}{l}\text { Fundación Ortega y Gasset- } \\
\text { Marañón }\end{array}$ & $\mathrm{X}$ & $\mathrm{X}$ & $\mathrm{X}$ & $\mathrm{X}$ & $\mathrm{X}$ & & & & 5 \\
\hline $\begin{array}{l}\text { Grupo de Estudios sobre } \\
\text { Política y Seguridad } \\
\text { Internacional (GESI) }\end{array}$ & $\mathrm{X}$ & $\mathrm{X}$ & $\mathrm{X}$ & $\mathrm{X}$ & $\mathrm{X}$ & & & & 5 \\
\hline IECAH & $\mathrm{X}$ & $X$ & $\mathrm{X}$ & $X$ & $\mathrm{X}$ & & & & 5 \\
\hline $\begin{array}{l}\text { Instituto Europeo del } \\
\text { Mediterráneo }\end{array}$ & $\mathrm{X}$ & $\mathrm{X}$ & & $\mathrm{X}$ & $\mathrm{X}$ & $\mathrm{X}$ & & & 5 \\
\hline $\begin{array}{l}\text { Instituto Gobernanza } \\
\text { Democrática }\end{array}$ & $\mathrm{X}$ & $\mathrm{X}$ & & $\mathrm{X}$ & & $\mathrm{X}$ & & Vimeo & 5 \\
\hline Instituto Juan de Mariana & $\mathrm{X}$ & $\mathrm{X}$ & $\mathrm{X}$ & $\mathrm{X}$ & $\mathrm{X}$ & & $\mathrm{X}$ & & 5 \\
\hline $\begin{array}{l}\text { Basque centre for climate } \\
\text { change }\end{array}$ & $\mathrm{X}$ & $\mathrm{X}$ & $\mathrm{X}$ & & & & & Vimeo & 4 \\
\hline $\begin{array}{l}\text { Centro de Desarrollo } \\
\text { Internacional, Universidad de } \\
\text { Navarra }\end{array}$ & & $\mathrm{X}$ & & $\mathrm{X}$ & & $\mathrm{X}$ & & & 4 \\
\hline Cercle per al Coneixement & $X$ & $X$ & $\mathrm{X}$ & & & & & Vimeo & 4 \\
\hline
\end{tabular}


RLCS, Revista Latina de Comunicación Social, 77, 253-273

[Investigación] DOI: 10.4185/RLCS-2020-1457 | ISSN 1138-5820 | Año 2020

\begin{tabular}{|c|c|c|c|c|c|c|c|c|c|}
\hline Ecodes & $\mathrm{X}$ & $\mathrm{X}$ & $\mathrm{X}$ & $\mathrm{X}$ & & & & & 4 \\
\hline FAES & $\mathrm{X}$ & $\mathrm{X}$ & $\mathrm{X}$ & $\mathrm{X}$ & & $\mathrm{X}$ & & & 4 \\
\hline Fedea & & $\mathrm{X}$ & $\mathrm{X}$ & $\mathrm{X}$ & & $\mathrm{X}$ & & Vimeo & 4 \\
\hline Fundación Independiente & $\mathrm{X}$ & $\mathrm{X}$ & $\mathrm{X}$ & $\mathrm{X}$ & & & $\mathrm{X}$ & & 4 \\
\hline Instituto Estudios Económicos & $\mathrm{X}$ & $\mathrm{X}$ & & $\mathrm{X}$ & $\mathrm{X}$ & & & & 4 \\
\hline $\begin{array}{l}\text { Asoc. de Investigación y } \\
\text { Especialización sobre Temas } \\
\text { Iberoamericanos }\end{array}$ & $\mathrm{X}$ & $\mathrm{X}$ & $\mathrm{X}$ & & & & & & 3 \\
\hline FUNCAS & & $\mathrm{X}$ & & $\mathrm{X}$ & $\mathrm{X}$ & $\mathrm{X}$ & & & 3 \\
\hline Fundación Cultura de Paz & $X$ & & & $\mathrm{X}$ & $\mathrm{X}$ & & & & 3 \\
\hline Fundación Euroamérica & $\mathrm{X}$ & $\mathrm{X}$ & $\mathrm{X}$ & & & & & & 3 \\
\hline $\begin{array}{l}\text { Fundación Iberoamérica y } \\
\text { Europa }\end{array}$ & $\mathrm{X}$ & $\mathrm{X}$ & $\mathrm{X}$ & & $\mathrm{X}$ & & & & 3 \\
\hline Fundación Pablo Iglesias & $X$ & $\mathrm{X}$ & & & & & & Vimeo & 3 \\
\hline GRAIN & $\mathrm{X}$ & $\mathrm{X}$ & $\mathrm{X}$ & & & & & & 3 \\
\hline $\begin{array}{l}\text { Grupo de Análisis sobre } \\
\text { Estrategia y Prospectiva de la } \\
\text { Información }\end{array}$ & $\mathrm{X}$ & $\mathrm{X}$ & & & & $\mathrm{X}$ & & & 3 \\
\hline $\begin{array}{l}\text { GEES (Grupo de Estudios } \\
\text { Estratégicos) }\end{array}$ & $\mathrm{X}$ & $\mathrm{X}$ & $\mathrm{X}$ & & & & & & 3 \\
\hline $\begin{array}{l}\text { Seminario de Investigación } \\
\text { para la Paz del Centro } \\
\text { Pignatelli }\end{array}$ & $\mathrm{X}$ & $\mathrm{X}$ & & $\mathrm{X}$ & & & & & 3 \\
\hline $\begin{array}{l}\text { Centro de Estudios Políticos y } \\
\text { Constitucionales }\end{array}$ & & $\mathrm{X}$ & $\mathrm{X}$ & & & & & & 2 \\
\hline Círculo de Economía & & $\mathrm{X}$ & & $\mathrm{X}$ & & $\mathrm{X}$ & & & 2 \\
\hline $\begin{array}{l}\text { Fundación de Investigaciones } \\
\text { Marxistas }\end{array}$ & $X$ & $\mathrm{X}$ & & & & & & & 2 \\
\hline Iberglobal & $\mathrm{X}$ & $\mathrm{X}$ & & & $\mathrm{X}$ & & & & 2 \\
\hline Instituto Estudios Fiscales & $\mathrm{X}$ & & & & $\mathrm{X}$ & & & & 2 \\
\hline $\begin{array}{l}\text { Observatorio Nacional de las } \\
\text { Telecomunicaciones y de la SI }\end{array}$ & & $\mathrm{X}$ & $\mathrm{X}$ & & & & & & 2 \\
\hline Fundación Sistema & $X$ & & & & & & & & 1 \\
\hline Instituto Estudios Democracia & $\mathrm{X}$ & & & & & & & & 1 \\
\hline $\begin{array}{l}\text { Instituto Universitario } \\
\text { Gutiérrez Mellado }\end{array}$ & $\mathrm{X}$ & & & & & & & & 1 \\
\hline $\begin{array}{l}\text { Centro Internacional de } \\
\text { Toledo para la Paz }\end{array}$ & & & & & & & & & 0 \\
\hline TOTAL & 41 & 42 & 31 & 30 & 22 & 19 & 11 & 13 & - \\
\hline PORCENTAJE (\%) & 85,41 & 87,5 & 64,58 & 62,5 & 45,83 & 39,58 & 22,91 & 27,08 & - \\
\hline
\end{tabular}

Fuente: elaboración propia.

Atendiendo al análisis desarrollado, se observa que en Facebook la actividad en la mayoría de los perfiles estudiados no es diaria, siendo la frecuencia media de actualización de contenido de 18 o 19 entradas al mes. Los centros que destacan por tener una mayor actividad son el Instituto Juan de Mariana, la Fundación Alternativas y la Fundación Independiente. En lo que respecta al contenido compartido, la media es de 2 entradas al mes. La Fundación Carolina encabeza el grupo con 35 entradas compartidas desde el perfil de Jesús Andreu, director de la fundación. En general, solo el $33,3 \%$ de los que utilizan Facebook han compartido contenido en sus cuentas en el periodo analizado. 
Por su parte, la actividad de los think tanks en Twitter es mayor que la registrada en Facebook. La media de contenido propio en las cuentas analizadas es de 63 o 64 entradas al mes. Resaltan las cuentas de la Fundación de la Innovación Bankinter, el Real Instituto Elcano y el Barcelona Institute for Global Health. Atendiendo al contenido compartido o retuiteado, la frecuencia media es de 33 o 34 entradas al mes, destacando las cuentas de la Fundación Ciudadanía y Valores, la Fundación Independiente y la Fundación Pablo Iglesias.

A pesar de que el 62,5\% de los think tanks poseen perfil en YouTube, la actividad registrada en las cuentas se puede calificar de escasa. La frecuencia media de actualización de contenido oscila entre 2 y 3 entradas al mes, destacando la actividad del Instituto Europeo del Mediterráneo, la Fundación de la Innovación Bankinter y el Círculo de Economía.

Como herramienta específica para la difusión de contenidos de carácter bidireccional, el 39,5\% de las organizaciones analizadas disponen de blogs. Dentro de este porcentaje se identifican algunos espacios desactualizados (ThinkEPI, Iberglobal, Fundación Ciudadanía y Valores e Institución Futuro), otros que no son blogs propios (Ecodes y el del Centro de Estudios Políticos y Constitucionales) y otros que no podrían considerarse una herramienta de comunicación bidireccional ya que no permiten comentarios (Círculo de Empresarios, FUNCAS y Fundación Ciudadanía y Valores).La frecuencia media de actualización del contenido en estas plataformas fluctúa entre 8 y 9 entradas al mes. Destaca la actividad diaria de GRAIN, que cuenta con 64 entradas en el intermedio analizado. En el extremo contrario se localiza un $12.65 \%$ de blogs desactualizados.

Finalmente, examinando la temática de las entradas, se puede determinar que en Facebook son los siguientes temas los que tienen una mayor presencia:

- Publicación de información de eventos propios o de otros centros afines con los que comparten intereses o a los que acuden, intervienen o apoyan.

- Información de estudios, informes, proyectos y publicaciones periódicas, así como convocatorias de becas y ayudas, cursos, másteres y doctorados.

- Entradas tematizadas en relación a la celebración de días conmemorativos y efemérides a nivel nacional o internacional.

- Finalmente, se comparten y difunden noticias nacionales e internacionales relacionadas con sus intereses, así como publicaciones en medios de comunicación; prevaleciendo la intervención de sus expertos en periódicos con artículos de opinión.

En Twitter, los temas tratados son los mismos que en Facebook, aunque se comparte más información de actos propios o de otros centros en los que intervienen y la retransmisión de los eventos es más exhaustiva.

En diversas ocasiones, el contenido compartido por estas cuentas es publicado por universidades a las que pertenecen estos grupos o con las que mantienen una estrecha relación. Es el caso de la Fundación Ciudadanía y Valores, que comparte publicaciones de la UNIR; la Fundación de Investigaciones Marxistas que da difusión a los cursos de verano de la Universidad Complutense de Madrid; el Centro de Desarrollo Internacional vinculado a la Universidad de Navarra; el Grupo de Estudios sobre Política y Seguridad Internacional (GESI) de la Universidad de Granada o el Instituto Estudios Democracia adscrito a la Universidad CEU San Pablo.

Los canales en YouTube son utilizados para subir vídeos en los que se retransmiten eventos, entre los que destacan presentaciones de libros, informes, proyectos y entrega de premios. Existen bastantes vídeos en los que se pueden ver charlas e intervenciones de expertos, así como entrevistas, como es 
el caso del espacio Diálogos FAES. Por otra parte, esta red se utiliza para presentar videos conmemorativos y presentaciones de documentales.

De forma particular, los blogs analizados permiten presentar a los expertos y equipos de investigación que forman parte de los think tanks, así como sus contribuciones en informes y estudios. Como elemento de apoyo se publican entrevistas a estos investigadores y expertos. En general, estos espacios proporcionan una información más ampliada de sus estudios e investigaciones a un público más interesado en sus temáticas.

\section{Discusión y conclusiones}

Con el estudio desarrollado se alcanza el objetivo general de estudiar cómo los think tanks españoles utilizan el medio digital para difundir su actividad y transmitir sus ideas. De manera específica, el trabajo permite conocer qué tipos de herramientas de comunicación emplean este tipo de entidades en los distintos espacios digitales que implementan de manera oficial (sedes webs y medios sociales) y el uso que realizan de las mismas. Del análisis se infiere una actividad difusora de las actividades realizadas por los think tanks sin incorporación de elementos discursivos y específicos para el entorno digital.

El ecosistema comunicativo digital permite que los think tanks puedan gestionar de una mejor manera a los diferentes públicos con los que se relacionan y especificar las herramientas a partir de las características sociocomunicativas de sus audiencias.

En las plataformas se identifican herramientas de carácter unidireccional y otras de tipo bidireccional. Las primeras contribuyen a la presentación de la identidad de estas organizaciones y a la difusión de sus actividades e ideas. Entre los recursos unidireccionales se localizan herramientas genéricas (agendas de actividades, difusión de informes o secciones de noticias) y otras de carácter específico destinadas a la comunicación con profesionales de la información (salas de prensa virtuales). Las herramientas bidireccionales, por su parte, se ocupan de la difusión interactiva de las actividades de los think tanks, cobrando una relevancia particular el empleo de medios sociales (redes sociales y blogs). Estos últimos recursos tratan de fomentar el compromiso con sus públicos (Castillo-Esparcia, García Ponce y Smolak, 2013; Coombs y Holladay, 2015; Gershon; 2016 y Kent, 2013).

En ambos casos (herramientas unidireccionales y bidireccionales) resulta de especial significación la función de educación, sensibilización y legitimación de ciertas posturas sociales apuntada anteriormente (Bürger, 2015; Carim y Warwick, 2013; Ciszek, 2016; Martínez-Salas y Campillo, 2018). Dicha función se lleva a cabo a través de dos vías: una más especializada que va dirigida a un público selectivo y otra que va destinada a un público más amplio con el objeto de mostrar o colocar determinadas temáticas con mayor importancia sobre otras. En esta estructuración de los temas, también tiene lugar determinados encuadres, en los que los think tanks tienen la oportunidad de presentar unas soluciones frente a otras posibles a dichos asuntos.

La función de educación se articula a través de institutos de investigación, universidades de mayores, programas de intercambio, escuelas de verano y numerosas actividades formativas. En esta línea se aprecia una clara vinculación de estas instituciones con diversos centros universitarios.

Los medios sociales mayoritariamente empleados son Twitter y Facebook, seguidos de LinkedIn y YouTube. Estas plataformas se utilizan prioritariamente para difundir contenidos propios. Atendiendo a su temática, los contenidos ofrecidos suelen girar en torno a tres ejes fundamentales: 
eventos, estudios (apoyados, en numerosas ocasiones, por charlas o entrevistas con expertos) y noticias. Twitter y YouTube suelen ser las plataformas más usadas para la retransmisión de eventos de manera más exhaustiva.

La mayor frecuencia de utilización de la plataforma de Twitter sobre Facebook nos puede indicar que, dada la utilización de la primera de ellas por parte de estas instituciones, prevalecen los flujos comunicativos unidireccionales o bidireccionales asimétricos, mientras que la segunda se emplea con un carácter más interactivo en el medio digital. Lo que puede traducirse en que todavía los think tanks muestran un mayor interés en establecer una relación comunicativa difusora o una bidireccionalidad asimétrica con sus diferentes públicos en lugar de la simétrica, en la que estos últimos tendrían mayores oportunidades de expresarse mostrando una iniciativa propia.

Finalmente, los blogs siguen siendo parte importante de la comunicación digital de los think tanks españoles, a pesar de que ya comienza a observarse un porcentaje significativo de cuentas desactualizadas. Su aprovechamiento se centra en la difusión detallada de contribuciones de expertos y equipos de investigación.

\section{Referencias}

Abelson, D. (2002). Do Think Tanks Matter? Opportunities, Constraints and Incentives for Think Tanks in Canada and the United States. Global Society, 14(2).

Abelson, D. (2012). Think Tanks, Social Democracy and Social Policy. Parliamentary Affairs, 66(4), 894-902.

Al-Deen, H. S. N., \& Hendricks, J. A. (2013). Social media and strategic communications. Palgrave MacMillan.

Almansa-Martínez, A., y Fernández-Torres, M. J. (2011). Estudio sobre la comunicación digital de las organizaciones sociales en España. Vivat Academia, (117), 337-252. http://dx.doi.org/10.15178/va.2011.117E.337-352

Auger, G. (2013). Fostering Democracy through Social Media: Evaluating Diametrically Opposed Non-Profit Advocacy Organizations' Use of Facebook, Twitter and YouTube". Public Relations Review, 39(4), 369-376.

Bürger, T. (2015). Use of Digital Advocacy by German Non-Profit Foundations on Facebook. Public Relations Review, 41(4), 523-525. https://doi.org/10.1016/j.pubrev.2015.07.007

Carim, L., \& Warwick, C. (2013). Use of social media for corporate communications by researchfunding organizations in the UK. Public Relations Review, (39), 521-525.

Castillo-Esparcia, A. (2009). Relaciones públicas y think tanks en América Latina. Estudio sobre su implantación y acción. Razón y Palabra, (70), 1-22.

Castillo-Esparcia, A.; García-Ponce, D.; y Smolak-Lozano, E. (2013). Movimientos sociales y estrategias de comunicación. El caso de 15-M y de Occupy Wall Street. Estudios Sobre El Mensaje Periodístico, 19(1), 71-89. 
RLCS, Revista Latina de Comunicación Social, 77, 253-273

[Investigación] DOI: 10.4185/RLCS-2020-1457 | ISSN 1138-5820 | Año 2020

Ciszek, E. (2016). Digital Activism: How social media and dissensus inform theory and practice. Public Relations Review, 42(2), 314-321. https://doi.org/10.1016/j.pubrev.2016.02.002

Coombs, T., \& Holladay, S. (2015). Public Relations 'relationships identity' in research: Enlightenment or Illusion. Public Relations Review, 41(5), 689-695.

https://doi.org/10.1016/j.pubrev.2013.12.008

Gershon, R. (2016). Digital Media and Innovation. Management and Design. Strategies in Communication. Sage.

Hallahan, K., Holtzhausen, D., van Ruler, B., Vercic, D., \& Sriramesh, K. (2007). Defining strategic communication. International Journal of Strategic Communication, 1(1), 3-35.

Jan MarkVerheyden, P. (2017). Knowing what is said on enterprise social media: towards the development of an analytical communication framework. Revista Internacional de Relaciones Públicas, VII(13), 5-22. http://dx.doi.org/10.5783/RIRP-13-2017-02-05-22

Kent, Michael (2013). Using social media dialogically: public relations role in reviving democracy. Public Relations Review, 39(4), 337-345. https://doi.org/10.1016/j.pubrev.2013.07.024

Lazuela, F., \& Girona, R. (2016). The impact of think tanks on mass media discourse regarding the economic crisis in Spain. Public Relations Review, 42(2), 271-278.

https://doi.org/10.1016/i.pubrev.2015.09.006

Martínez-Sala, A. M., y Campillo-Alhama, C. (2018). La gestión de las redes sociales turísticas desde la perspectiva de las relaciones públicas 2.0: la importancia del diálogo. Revista Internacional de Relaciones Públicas, VIII(16), 5-26. http://dx.doi.org/10.5783/RIRP-16-2018$\underline{02-05-26}$

McGann, J., \& Weaver, K. (2000). Think-tanks and Civil Societies, catalysts for Action. New Brunswick, Transaction Publishers.

McGann, J. (2011). Global Go To Think Tank Index Report. University of Pennsylvania.

McGann, J.; Viden A., \& Rafferty, J. (2014). How Think Tanks Shape Social Development Policies. Pennsylvania University Press.

McGann, J. (2017). Global Go To Think Tank Index Report.

https://repository.upenn.edu/cgi/viewcontent.cgi?article=1012\&context=think_tanks

Misztal, Barbara (2012). Public intellectuals and think tanks: A free market in ideas? International Journal of Politics, Culture and Society, 25(4), 127-141.

Rich, A., \& Weaver, K. (2000). Think tanks in the US media. The International Journal of Press/Politics, 5(4), 81-103. https://doi.org/10.1177/1081180X00005004006

Stone, D. (1996). Capturing the Political Imagination: Think Tanks and the Policy Process. Frank Cass. 
RLCS, Revista Latina de Comunicación Social, 77, 253-273

[Investigación] DOI: 10.4185/RLCS-2020-1457 | ISSN 1138-5820 | Año 2020

Stone, D., \& Denham, A. (2004). Think Tank Traditions: Policy Research and the Politics of Ideas. Manchester University Press.

Tello, M. (2013). Guía de Think tanks en España. Segunda edición. UNED.

Valentini, C. (2015). Is using social media good for the public relations profession? A critical reflection. Public Relations Review, 41(2), 170-177. https://doi.org/10.1016/j.pubrev.2014.11.009

Xifra, J. (2003). Teoría y estructura de las relaciones públicas. McGraw-Hill.

\section{AUTORES:}

\section{Antonio Castillo-Esparcia}

Coordinador del Máster Oficial "Dirección Estratégica e Innovación en Comunicación”, es Doctor y Licenciado por la Universidad Autónoma de Barcelona. Autor de más de 150 publicaciones nacionales e internacionales sobre relaciones públicas, comunicación estratégica, lobbies y think tanks. Codirector del Proyecto de I+D "Lobby y comunicación". Profesor visitante en universidades europeas y americanas. Presidente de la Asociación de Investigadores en Relaciones Públicas.

acastilloe@uma.es

Orcid ID: https://orcid.org/0000-0002-9751-8628

Google Scholar: https://scholar.google.com/citations?user=b541fg8AAAAJ\&hl=en

\section{Elizabet Castillero-Ostio}

Doctora en Ciencias de la Comunicación por la Universidad de Málaga. Licenciada en Publicidad y Relaciones Públicas y Máster en Gestión Estratégica e Innovación en Comunicación. Técnico en Organización de Congresos y Eventos. Sus principales líneas de investigación giran en torno a la Comunicación Política e Institucional y al Protocolo y la Organización de Eventos, temáticas sobre las que ha publicado diversos artículos en revistas científicas, capítulos de libros, así como comunicaciones en congresos internacionales.

ecastillero@uma.es

Orcid ID: https://orcid.org/0000-0002-0546-7262

Google Scholar: https://scholar.google.es/citations?user=iSBFgbEAAAAJ\&hl=es\&oi=ao

\section{Ana Castillo-Díaz}

Coordinadora del Grado de Publicidad y Relaciones Públicas. Entre 2004 y 2015 ejerció su actividad docente e investigadora en la Universidad de Extremadura. Ha realizado estancias docentes y de investigación en diversas universidades europeas y americanas. Sus principales líneas de investigación se vinculan con la comunicación estratégica empresarial e institucional. En este campo cuenta numerosas publicaciones nacionales e internacionales.

anacastillo@uma.es

Orcid ID: https://orcid.org/0000-0002-3650-2964

Google Scholar: https://scholar.google.es/citations?user=YVlH8CIAAAAJ\&hl=es\&oi=ao 